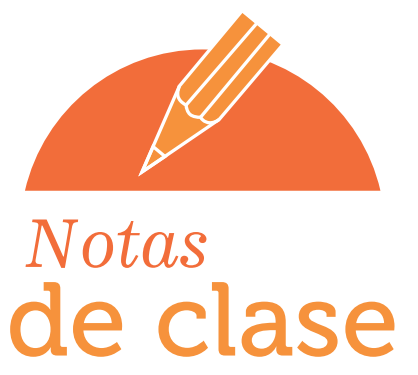

Apropiación social del conocimiento

\title{
ESTIMULACIÓN TÁCTIL KINESTÉSICA: ABORDAJE DESDE ENFERMERÍA
}

Nini Quintero Ramírez

Yuris Sánchez García

Yasmina Toncel

Liliana Reales Hernández

Universidad Cooperativa de Colombia

Sede Santa Marta 


\section{ACERCA DE LAS AUTORAS}

Nini Quintero Ramírez, magíster en Salud Ocupacional y Seguridad Industrial, profesor instructor, Universidad Cooperativa de Colombia, sede Santa Marta.

Correo-e: nini.quintero@campusucc.edu.co

orcid: https://orcid.org/0000-0001-6870-2664?

lang=en

Yuris Sánchez García, magíster en Salud Ocupacional y Seguridad Industrial, profesor instructor, Universidad Cooperativa de Colombia, sede Santa Marta.

Correo-e: yuris.sanchezg@campusucc.edu.co orcid: https://orcid.org/0000-0002-4074-6240

Yasmina Toncel, magíster en Desarrollo Integral en Niño y Adolescente, profesor auxiliar, Universidad Cooperativa de Colombia, sede Santa Marta.

Correo-e: yasmina.toncel@campusucc.edu.co orcid: https://orcid.org/0000-0001-7913-0171

Liliana Reales Hernández, magíster en Ciencias de la Enfermería, profesor aspirante especialista, Universidad Cooperativa de Colombia, sede Santa Marta.

Correo-e: liliana.reales@campusucc.edu.co

orcid: https://orcid.org 0000-0001-9396-0534

\section{CÓMO CITAR ESTE DOCUMENTO}

Quintero Ramírez N, Sánchez García Y, Toncel Y, Reales Hernández L. Estimulación táctil kinestésica: abordaje desde enfermería. (Generación de contenidos impresos $\mathrm{N}^{\circ} 5$ ). Ediciones Universidad Cooperativa de Colombia, 2021. doi: https://doi.org/10.16925/gcnc.17

\section{NOTA LEGAL}

El presente documento de trabajo ha sido incluido dentro de nuestro repositorio institucional como Apropiación social de conocimiento por solicitud del autor, con fines informativos, educativos o académicos. Asimismo, los argumentos, datos y análisis incluidos en el texto son responsabilidad absoluta del autor y no representan la opinión del Fondo Editorial o de la Universidad.

\section{DISCLAIMER}

This coursework paper has been uploaded to our institutional repository as Social Appropriation of Knowledge due to the request of the author. This document should be used for informational, educational or academic purposes only. Arguments, data and analysis included in this document represent authors' opinion not the Press or the University.

(c) (1) Este documento puede ser consultado, descargado o reproducido desde nuestro repositorio institucional (http://repository.ucc.edu.co/handle/20.500.12494/7369) para uso de sus contenidos, bajo la licencia de Creative Commons Reconocimiento-NoComercial-SinObraDerivada 4.0 Internacional. http://creativecommons.org/licenses/by-nc-nd/4.0/ 


\section{TABLA DE CONTENIDO}

INTRODUCCIÓN

UNIDAD 1

Definiciones 6

UNIDAD 2

Principios básicos para la elección de la técnica 6

Signos de estrés $\quad 6$

UNIDAD 3

Técnica para la realización del masaje kinestésico 7

UNIDAD 4

Intervención de enfermería durante el estímulo 8

táctil kinestésico en el recién nacido prematuro

CONCLUSIONES 9

$\begin{array}{lr}\text { REFERENCIAS } & 9\end{array}$ 


\title{
05 ESTIMULACIÓN TÁCTIL KINESTÉSICA: ABORDAJE DESDE ENFERMERÍA
}

\author{
Nini Quintero Ramírez \\ Yuris Sánchez García \\ Yasmina Toncel \\ Liliana Reales Hernández
}

\section{Resumen}

La supervivencia de neonatos que no cumplen su desarrollo gestacional completo ( $<34$ semanas) se ve sometida a técnicas que estimulan su desarrollo neuronal. Por lo tanto, se da a conocer la técnica de estimulación táctil kinestésica, que consiste en desarrollar los estímulos sensoriales del neonato; así mismo, se describe cómo llevar a cabo ese proceso y el aprendizaje adquirido por los neonatos. El presente documento hace un abordaje grosso modo de la técnica de estimulación táctil kinestésica de acuerdo con algunos autores que han investigado acerca de la temática.

Palabras clave: estimulación, intervenciones, neurodesarrollo, enfermería, kinestésica. 


\section{INTRODUCCIÓN}

En países desarrollados, se evidencia que actualmente por lo menos entre 8 y $10 \%$ de los nacidos vivos solo alcanzan las 37 semanas de gestación. A pesar de que el proceso gestacional normal de una mujer es de 40 semanas, en algunos casos suele interrumpirse ya sea por circunstancias biológicas o psicológicas, alteraciones de la salud de la madre, deficiencia del desarrollo embrionario del feto o alteraciones ambientales. Sin embargo, un alto número de recién nacidos pretérmino permanece debido a las intervenciones brindadas mediante el periodo crítico de adaptación al medio extrauterino [1].

La expectativa de vida de los recién nacidos prematuros lastimosamente tiene relación con el lugar de nacimiento. Acorde con los datos consultados, se estima que solo subsiste el $10 \%$ de los prematuros extremos, cuya consecuencia es el nacimiento de un neonato prematuro, que es aquel que nace con menos de 34 semanas, son considerados inmaduros neurológicamente y no solo deben recibir asistencia médica, sino que además el estrés al que son sometidos, por ambientes agresivos y su poco desarrollo, logra ser la disrupción entre la relación padres-hijo y alterar su desarrollo físico y emocional [2, 3].

Se evidencia que, entre las primeras causas de mortalidad de los neonatos en el primer mes de vida, debido a su dificultad para adaptarse al medio extrauterino, están los medios ambientales agresivos, los procedimientos y el estrés. Molano [2] lo detalla es su estudio. Sin embargo, existen intervenciones específcas en el cuidado de los neonatos prematuros, al igual que avances científicos y tecnológicos que aumentan la supervivencia de los neonatos. Es el caso de la aplicación del método madre canguro y de la técnica de estimulación táctil kinestésica, cuya finalidad se fundamenta en el progreso de las destrezas del cuerpo del recién nacido, al igual que su sistema sensorial, motriz, propioceptivo y cognitivo, el contacto piel a piel, los beneficios neurológicos, la regulación de la temperatura corporal y el ritmo de ganancia de peso, lo que lleva a disminuir los riesgos de complicaciones y de infecciones [2, 4].

Se trata de un proceso que contribuye a la formación del recién nacido a través de su expresión corporal, impulsando a los órganos motores a tener reacciones espontáneas; por consiguiente, se considera como la complementación del desarrollo gestacional normal de un individuo. El presente texto hace un abordaje de la estimulación táctil kinestésica, su aplicación y los beneficios que aporta a los neonatos $[5,6]$.

Este material de consulta es de gran importancia para los estudiantes del curso "Cuidado de Enfermería al Niño y al Adolescente”, en especial durante el desarrollo de las prácticas formativas realizadas en el servicio de Unidad de Cuidados Intensivos (UCI) neonatal.

El neonato prematuro hospitalizado debe ser sometido a cuidados y procedimientos que ayuden a mejorar movimientos espontáneos en todos sus sistemas con el medio que los rodea. Partiendo de esta necesidad, se somete a estimulación táctil kinestésica.

En la actualidad, las intervenciones que van encaminadas a mejorar la supervivencia de los neonatos han dado como resultado ganancia de peso, menor estancia hospitalaria y vínculo afectivo con la madre [7].

Por lo anterior, el presente documento aborda aspectos relacionados con la aplicación de técnica kinestésica y la supervivencia de los neonatos, pasando por las intervenciones aplicadas por el personal profesional de enfermería.

Se recomienda entonces que los estudiantes se apropien del contenido del siguiente 
documento, y lo analicen y debatan de manera organizada, antes de enfrentarse a la práctica para que adquieran confianza y seguridad en sus encuentros con el neonato y el cuidador durante la aplicación de las intervenciones.

\section{UNIDAD 1}

\section{DEFINICIONES}

Estimulación: la estimulación temprana permite potencializar el buen desarrollo físico, social, cognitivo y emocional de los niños y las niñas durante los primeros años. En esta etapa, se potencializan todas las habilidades y capacidades a través de técnicas e intervenciones, y por ello es importante que se establezcan lazos de afectos y amor entre los padres y/o cuidadores como es abrazar, felicitar, sonreír mirar, tocar, hablar, con lo que se contribuye al desarrollo pleno, armonioso y seguro.

Kinestésica: la cinestesia es la ciencia que estudia el movimiento humano o la sensación de la percepción como movimiento universal. Esta herramienta se usa para sustituir o potenciar las funciones corporales relacionadas con propiocepción.

La inteligencia kinestésica: está asociada con la capacidad para controlar nuestro cuerpo en actividades físicas coordinadas como prácticas deportivas, el baile y las destrezas manuales. Mediante la inteligencia kinestésica corporal obtenemos información que, por efecto del movimiento y la vivencia, se transforma en aprendizaje significativo.

Estímulo externo: agente exterior que tiene la capacidad de inducir una reacción en el recién nacido hospitalizado. Tienen que ver con cambios físicos, químicos, mecánicos o de otra índole, y de esta forma atraen a los receptores. De ahí que puedan transferir esta intuición al sistema nervioso de los seres vivos, estableciendo una búsqueda, y liberar en ellos una respuesta [7].
Neurodesarrollo: suceso en el cual participan aspectos biológicos y del entorno que están frecuentemente interrelacionados. Favorecen la maduración de organizaciones nerviosas que responden a la práctica, dando como resultado aprendizajes cognitivos y de desarrollo físico [8].

\section{UNIDAD 2}

\section{PRINCIPIOS BÁSICOS PARA LA ELECCIÓN DE LATÉCNICA}

- Factores intrínsecos

- Elección de la técnica

- Duración

- Frecuencia

- Posición: observación y escucha de parámetros

- Considerar los signos de estrés

\section{SIGNOS DE ESTRÉS}

- Piel moteada

- Frecuencia de hipo

- Fascia roja

- Ceño fruncido

- Nariz pálida

- Piel reticulada

- Movimientos repetitivos de brazos y manos

- Posición de sandifel

- Caída de la saturación

- $\quad$ Llanto

- Bostezo

- Rigidez de los brazos y las piernas

- Desviación de la mirada hacia el estímulo

- Irritabilidad

- Aumento de la frecuencia cardiaca

- Aumento de la frecuencia respiratoria 


\section{UNIDAD 3}

\section{TÉCNICA PARA LA REALIZACIÓN DEL MASAJE KINESTÉSICO}

La estimulación táctil kinestésica consiste en una primera fase de masajeo y en segunda fase de estimulación kinestésica. La estimulación táctil se realiza preferiblemente en un colchón de agua (también llamado "vestíbulo") a través de seis pasos:
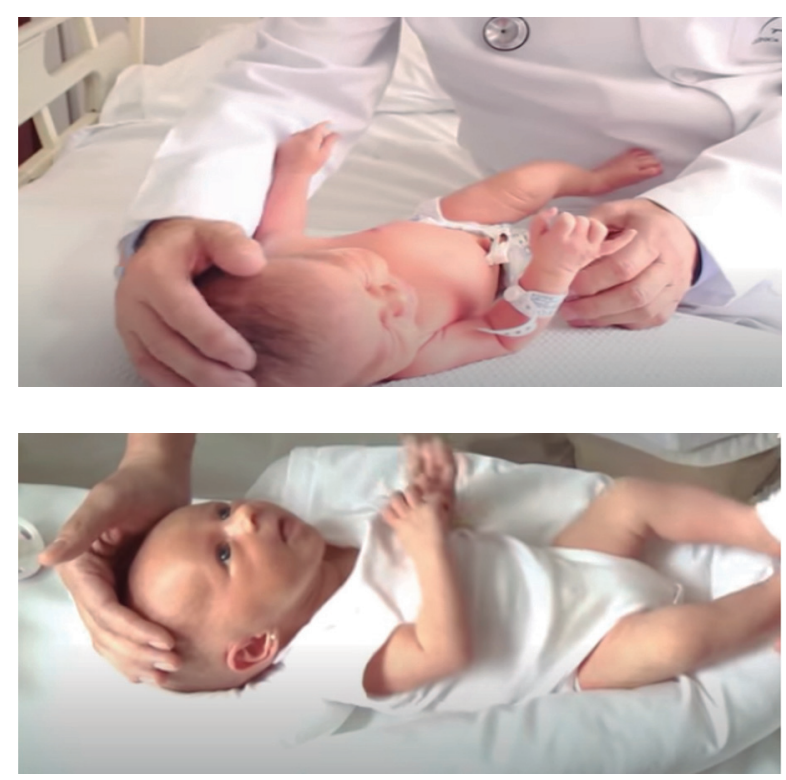

Figura 1. Primer paso. La cara desde la coronilla hasta el cuello.

Fuente: https://youtu.be/DcmMIOiYICk

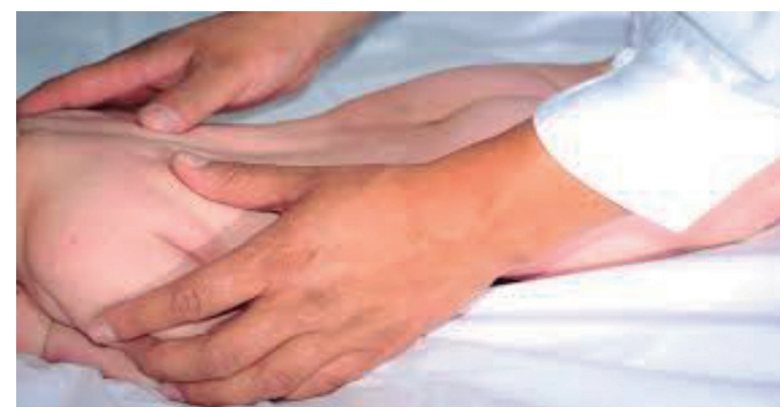

Figura 2. Segundo paso. En los hombros desde la espalda hasta los brazos.

Fuente: https://youtu.be/iSsFU460pqQ

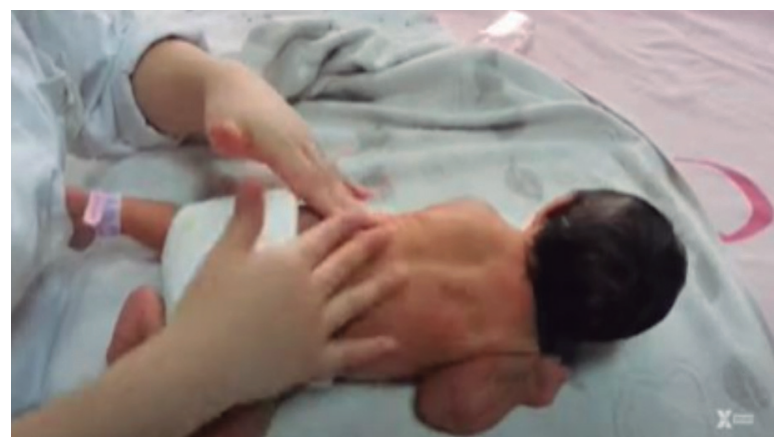

Figura 3. Tercer paso. Desde el cuello hasta la cintura. Fuente: https://www.youtube.com/watch?v=LhaXOCqY-Oc

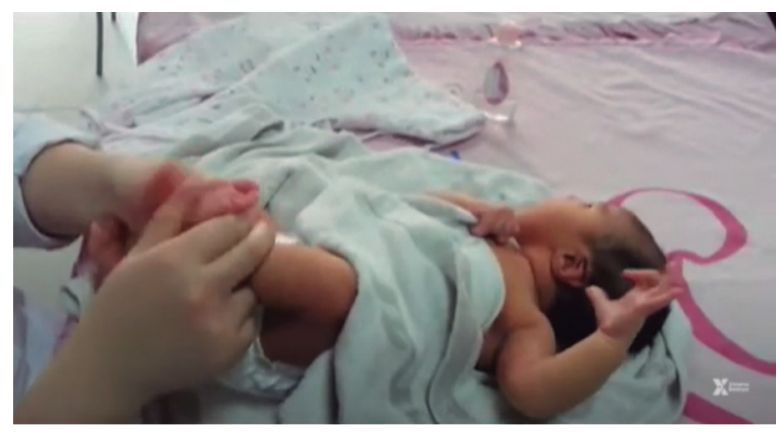

Figura 4. Cuarto paso. En las extremidades inferiores desde la parte superior de los muslos hasta los tobillos.

Fuente: https://youtu.be/iSsFU460pqQ

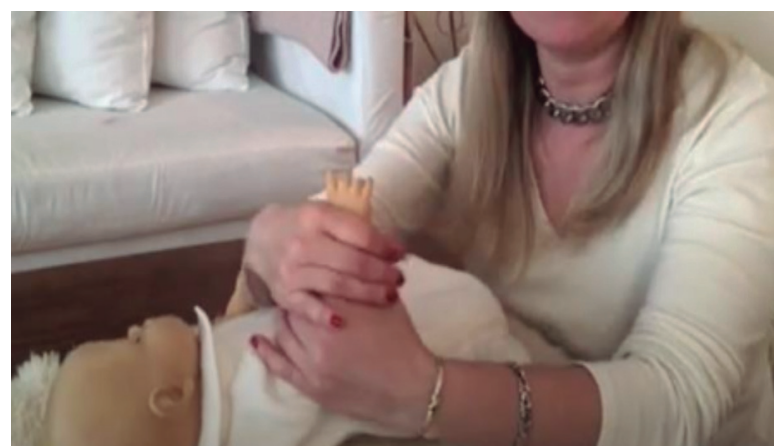

Figura 5. Quinto paso. En las extremidades superiores desde los hombros hasta las muñecas.

Fuente: https://www.youtube.com/watch?v=Q70RaAAmtJI

Por último, se pasa a decúbito supino y se le realizan seis movimientos de flexoextensión de cada extremidad superior e inferior a través del codo y de la rodilla, respectivamente, y también de las dos extremidades inferiores a la vez, durante diez minutos de tres y cinco veces al día [8]. 


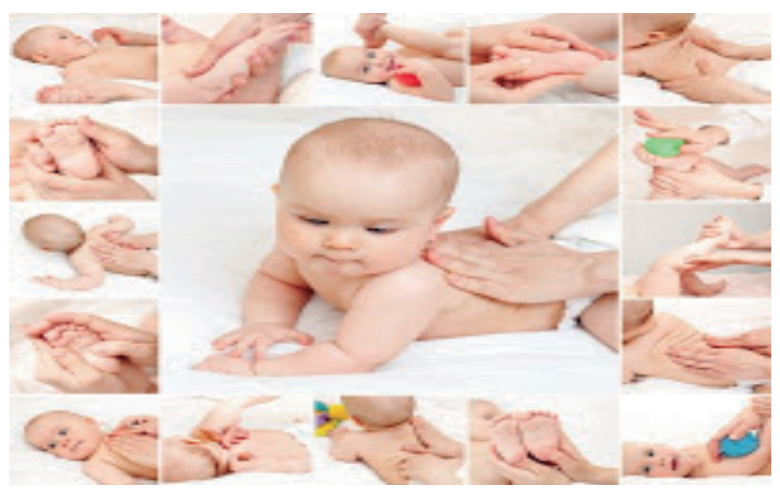

Figura 6. Sexto paso. Movimientos de flexoextensión de cada extremidad superior e inferior.

Fuente: http://www.origins.com/

En la conexión entre el prematuro y la madre se observará un avance favorable, resaltando la importancia entre el vínculo madre e hijo. Por consiguiente, al neonato se le facilita la alimentación independiente eficaz y más segura, ya que debe alcanzar la estabilidad fisiológica durante su proceso hospitalario [5, 3].

Las unidades investigativas han venido reflejando signos positivos gracias al trabajo en equipo de enfermeras, el equipo asistencial y los padres de los neonatos, lo que muestra una mayor confianza de los profesionales [1].

En este sentido, se manifiesta que el logro que alcanzan los neonatos al obtener respuestas positivas como el fortalecimiento de sus capacidades y la disminución del estrés prenatal depende del control pertinente por parte del personal asistencial encargado [6].

\section{UNIDAD 4}

\section{INTERVENCIÓN DE ENFERMERÍA DURANTE EL ESTÍMULO TÁCTIL KINESTÉSICO EN EL RECIÉN NACIDO PREMATURO}

El profesional de enfermería realiza modalidades de participación para optimizar la calidad de vida de los neonatos. Es por ello que respecto a la práctica de la estimulación táctil kinestésica genera una respuesta fisiológica y comportamental en la que se disminuye el estrés del recién nacido pretérmino, utilizando como referencia masajes, posición prona, toques, método canguro, estímulos de succión y la ecología neonatal [2].

El uso del masaje utilizando las manos, al igual que el contacto directo, genera un estímulo reconstituyente y reduce el estrés en el recién nacido pretérmino. El toque suave puede ser un bálsamo para cuando los neonatos están álgidos, o expuestos a estímulos dolorosos o a lugares hostiles, y su resultado es la disminución en la actividad motora, el alivio del llanto y la mejora de los tiempos de descanso [4].

Así mismo, el recién nacido pretérmino presenta como principal problema la enfermedad de membrana hialina. Por consiguiente, la posición mejora la oxigenación en neonatos con dificultad respiratoria y que reciben tratamiento con ventilación mecánica, pues requieren una menor fracción inspirada de oxígeno [9].

El tacto con tacto es de vital importancia para los recién nacidos que padecen situaciones estresantes y necesitan superarlas. Los efectos que presenta son crecimiento y desarrollo neurológico, ganancia de peso, apego, regulación fisiológica y adaptación [10].

Este tipo de técnica ha sido puesta en marcha por diferentes disciplinas de enfermería, evidenciando grandes beneficios para el neonato. Por eso se hace necesario fundamentarla con teorías de enfermería que apoyen las intervenciones realizadas por el profesional, como sería de interés en este caso el modelo de adaptación de Callista Roy. Hay que tener en cuenta que para conseguir el nivel deseado en el individuo, se debe responder a: los estímulos focales, que generarían una reacción interna e inmediata del neonato ante el tacto; el estímulo contextual, que está presente en las situaciones que lo rodean y contribuyen al 
efecto deseado del estímulo focal; y por último, el estímulo residual, que serían los factores más próximos del entorno que de una u otra manera pueden o no influir en el efecto deseado y que pueden ser controlados. También, se requiere lograr un proceso de afrontamiento y de mecanismos que, por medio de esta técnica, contribuyen al mejoramiento de la condición de salud del neonato [11].

\section{CONCLUSiOnes}

La estimulación táctil kinestésica integra los sentidos del neonato y facilita que sus funciones motoras tengan reacciones espontáneas.

El desarrollo de habilidades sensoriales, motrices, propioceptivas y cognitivas en neonatos menores de 36 semanas debe ser tratado con presencia de especialistas que induzcan a la madre a mejorar el estrés neuronal del neonato, con el fin de disminuir el grado de mortalidad.

Trasladar los conocimientos que se tengan en la actualidad para prevenir alteraciones posnatales y para que los neonatos maduren todo su sistema y crezcan de una manera eficaz.

A su vez, los estudiantes de séptimo semestre deben aplicar las técnicas aprendidas en los servicios de neonatos al realizar sus prácticas formativas en las unidades de cuidados intensivos, teniendo como referente una teoría de enfermería.

\section{REFERENCIAS}

Pacherrez Mamani IB, Condori López, LC, Palacios Torres ME, Medina Palacios SS. Influencia del método mamá canguro en el neurodesarrollo del recién nacido prematuro de 34 a 36 semanas en la unidad de cuidados intensivos neonatales de la Clínica Ricardo Palma. Lima: Universidad Peruana; 2017. https://hdl.handle.net/20.500.12866/797

Molano Pirazán ML. Efecto del Estímulo Táctil Kinestésico en la respuesta al estrés del recién nacido pretérmino en la Unidad de Cuidado Neonatal. Tesis doctoral. Universidad Nacional de Colombia; 2014. https://core.ac.uk/download/pdf/77269904.pdf

Cabezas Cardeñosa C, Marcos Montserrat, A. La importancia del entorno en los recién nacidos prematuros durante el ingreso hospitalario. Ágora de Enfermería. 2010;14(1):9-16. https://dialnet.unirioja.es/servlet/ articulo? codigo $=3666165$

Lizarazo Medina JP, Ospina Díaz JM, Ariza Riaño NE. Programa madre canguro: una alternativa protección de los recién nacidos prematuros o con bajo peso al nacer. Rev Salud Pública. 2012;14(2):32-45. http://www.scielo.org. $\mathrm{co/pdf} / \mathrm{rsap} / \mathrm{v} 14 \mathrm{~s} 2 / \mathrm{v} 14 \mathrm{~s} 2 \mathrm{a} 04 . \mathrm{pdf}$

Beltrán Ponce ZA. Influencia de la percepción kinestésica en el aprendizaje significativo de los niños de 4 a 5 años del Centro de Desarrollo Infantil Municipal María Olimpia Gudiño, en la ciudad de Ibarra. Trabajo de grado. Universidad Técnica del Norte; 2015. http://repositorio.utn.edu.ec/handle/123456789/4267

Chiliquinga Espinosa LV. Juegos al aire libre como estrategia de estimulación para la inteligencia kinestésica en los niños y niñas de 2 a 3 años en la Modalidad Creciendo con Nuestros Hijos en los sectores de San Cayetano, Mariano Acosta y Pugacho de la ciudad de Ibarra. Trabajo de grado. Universidad Técnica del Norte; 2015-2016. http://repositorio.utn.edu.ec/handle/123456789/5395

Gómez Coca S, Villamor Ruiz, EM, Ortiz Fernández S. Evidencia científica de la terapia kinestésica y su aplicación en prematuros. Trances: Transmisión del Conocimiento Educativo y de la Salud. 2017;9(6):909-920. https://dialnet.unirioja.es/servlet/articulo?codigo=6431726 
Sánchez AJ. Fisioterapia manual para favorecer el neurodesarrollo en bebés pretérmino estables en la Unidad de Cuidados Intensivos Neonatales. Trabajo de grado. Universidad de Navarra; 2016. https://academica-e. unavarra.es/xmlui/handle/2454/21183

Escobedo Chávez E, Montesino Vásquez C, Flores Nava G, López Padilla M. Posición prona para mejorar la oxigenación en neonatos con dificultad respiratoria sometido a ventilación mecánica. Rev Mex Pediatr. 1998;65(1):5-8.

Corporación del Prematuro Necocosur Chile. Estimulación táctil-kinestésica en RNPrT en UCIN (amor, tacto y nacimiento sensorial). Red de Salud UC-Christus; 2014.

Raile Alligood M. Modelos y teorías de enfermerías en enfermería. 9. a edición. Madrid: Elsevier; 2018. 


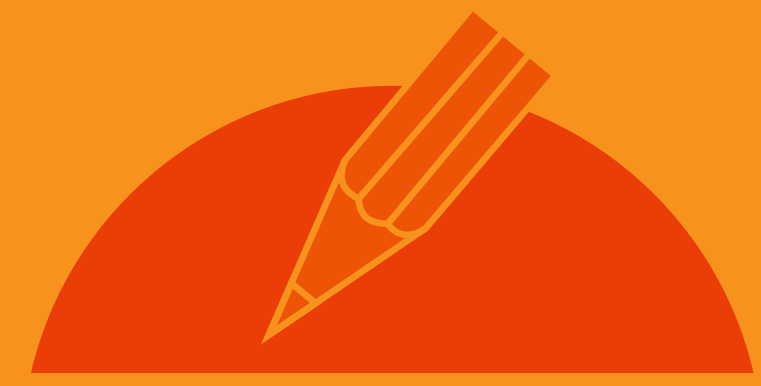

Notas

de clase 